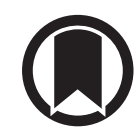

CrossMark

\title{
Airflow limitation by the Global Lungs Initiative equations in a cohort of very old adults
}

\author{
Eralda Turkeshi ${ }^{1}$, Bert Vaes ${ }^{1,2}$, Elena Andreeva ${ }^{1}$, Catharina Mathei ${ }^{1,2}$, \\ Wim Adriaensen $^{1,2}$, Gijs Van Pottelbergh ${ }^{1,2}$ and Jean-Marie Degryse ${ }^{1,2}$ \\ Affiliations: \\ ${ }^{1}$ Institute of Health and Society, Université Catholique de Louvain (UCL), Brussels, Belgium. \\ ${ }^{2}$ Dept of Public Health and Primary Care, Katholieke Universiteit Leuven (KUL), Leuven, Belgium.
}

Correspondence:

Eralda Turkeshi, Clos Chapelle-aux-Champs, 30 bte 31.15, 1200 Brussels, Belgium.

E-mail: eralda.turkeshidastudent.uclouvain.be

ABSTRACT The cut-off for forced expiratory volume in $1 \mathrm{~s}$ (FEV1)/forced vital capacity (FVC) defining airflow limitation for chronic obstructive pulmonary disease (COPD) is still contested. We assessed airflow limitation prevalence by the lower limit of normal (LLN) of Global Lungs Initiative (GLI) 2012 reference values and its predictive ability for all-cause mortality and hospitalisation in very old adults (aged $\geqslant 80$ years) compared with the fixed cut-off.

In a Belgian population-based prospective cohort of 411 very old adults, airflow limitation prevalence by the 5th percentile of GLI 2012 z-scores (GLI-LLN) and fixed cut-off (0.70) were compared with COPD reported by general practitioners (GPs). Survival and Cox regression multivariable analysis assessed the association of airflow limitation by both cut-offs with 5 -year all-cause mortality and first hospitalisation at 3 years.

9.2\% had airflow limitation by GLI-LLN and 27\% by fixed cut-off, without good agreement (kappa coefficient $\leqslant 0.40)$ with GP-reported COPD $(9 \%)$. Only airflow limitation by GLI-LLN was independently associated with mortality (adjusted hazard ratio 2.10 , 95\% CI 1.30-3.38). FEV1/FVC $<0.70$ but $\geqslant$ GLI-LLN (17.8\%) had no significantly higher risk for mortality or hospitalisation.

In a cohort of very old adults, airflow limitation by GLI-LLN has lower prevalence than by fixed cut-off, independently predicts all-cause mortality and does not miss individuals with significantly higher all-cause mortality and hospitalisation.

$@$ ERSpublications

Airflow limitation by GLI 2012 equations is better at predicting survival than fixed ratio in very old adults http://ow.ly/K0xMi

Editorial comment in Eur Respir J 2015; 46: 8-10 [DOI: 10.1183/09031936.00042215]

Received: Nov 242014 | Accepted after revision: Feb 182015 | First published online: April 162015

Conflict of interest: Disclosures can be found alongside the online version of this article at erj.ersjournals.com

Support statement: The BELFRAIL study (B40320084685) was supported by an unconditional grant from Fondation Louvain, Brussels, Belgium. Fondation Louvain is the support unit of Université Catholique de Louvain and is charged with developing the educational and research projects of the university by collecting gifts from corporations, foundations and alumni. Funding information for this article has been deposited with FundRef.

Copyright @ERS 2015 


\section{Introduction}

Chronic obstructive pulmonary disease (COPD) is projected to become an important cause of morbidity and mortality, especially due to worldwide population ageing [1]. Its diagnostic hallmark is irreversible airflow limitation based on the ratio of forced expiratory volume in $1 \mathrm{~s}$ (FEV1) to forced vital capacity (FVC), yet its cut-off and the definition of COPD diagnosis are still contested $[2,3]$.

Both the Global Initiative for Chronic Obstructive Lung Disease and the American Thoracic Society (ATS)/European Respiratory Society (ERS), in their guidelines for COPD diagnosis and treatment, recommend the use of the fixed cut-off of 0.70 to define airflow limitation [2, 4]. Yet, in their guidelines for the interpretation of lung function tests, ATS/ERS support the use of the lower limit of normal (LLN) at the 5th percentile of the frequency distribution of values of a reference "healthy" never-smoker population of equivalent age and sex. The fixed cut-off approach leads to overdiagnosis of COPD in older adults, as it does not take into account the age-related decline in respiratory parameters, while the LLN cut-off approach is dependent on age-specific reference values $[5,6]$. These have been lacking for adults aged $>80$ years or have been extrapolated from younger populations, until recently when the Global Lungs Initiative (GLI) all-age reference equations for different ethnic groups and populations aged 3-95 years were made available [5, 7]. The Lambda Mu Sigma (LMS) approach of calculating the LLN that uses the 5 th percentile of the distribution of $\mathrm{z}$-scores (similar to growth charts and bone mineral density) is considered more appropriate, as it takes into account the age-related decline in FEV1/FVC, variability of spirometry values and skewness of reference data $[5,8,9]$. It has also been found to be clinically valid based on its association with all-cause mortality, respiratory symptoms, incident mobility disability, COPD-related hospitalisations and FEV1 decline [10-12]. However, previous studies on the LMS approach of calculating the LLN did not use the GLI 2012 reference equations or focus on people aged $\geqslant 80$ years, the fastest growing age group among older adults $[10,13]$.

This study will compare the prevalence of airflow limitation based on the 5th percentile of GLI 2012 $z$-scores distribution (GLI-LLN) and the fixed cut-offs and their agreement with the COPD diagnosis reported by the general practitioner (GP) in a population-based cohort of very old adults (aged $\geqslant 80$ years). It will also evaluate and compare the predictive ability of airflow limitation defined by either/both cut-offs for all-cause mortality and first unplanned hospitalisation.

\section{Methods}

\section{Study design and population}

The BELFRAIL study $\left(\mathrm{BF}_{\mathrm{C} 80+}\right)$ is a prospective, observational, population-based cohort study of people aged $\geqslant 80$ years living in Belgium. Its aim is to improve the understanding of the epidemiology and pathophysiology of chronic diseases in this age group. The study protocol and sampling methods have already been described in detail [14]. Briefly, between November 2008 and September 2009, in 29 GP centres, 567 community-dwelling individuals aged $\geqslant 80$ years were recruited, excluding only those with known severe dementia, in palliative care and medical emergencies (acute illnesses needing urgent hospitalisation). At baseline, the participants' GPs recorded sociodemographic data and medical history. An extensive standardised assessment by a clinical research assistant included spirometry and blood sample collection. Hospitalisation data were collected from the GPs until $2.93 \pm 0.21$ years and mortality data until $5.19 \pm 0.22$ years from baseline. The study protocol was approved by the Biomedical Ethics Committee of the Medical School of the Universite Catholique de Louvain (UCL) in Brussels, Belgium, and all participants gave informed consent.

\section{Baseline spirometry}

All spirometry tests were administered by two trained clinical research assistants using a Spirobank spirometer (MIR, Rome, Italy) that has been found to be reliable for research purposes [15]. After demonstration of the correct spirometry manoeuvre, participants performed up to eight FVC manoeuvres or until exhaustion. Reversibility testing was not done. Repeatability of the spirometry was automatically calculated in accordance with ATS/ERS criteria [16]. Two independent researchers evaluated all spirograms by the ATS/ERS criteria and classified them in the following levels: 1) all acceptability and repeatability criteria were fulfilled; 2) all criteria were fulfilled except duration of expiration $>6 s$; 3) spirograms had good starts and no cough during the $1 \mathrm{~s}$ of manoeuvre; 4) none of the criteria were fulfilled. Participants with spirograms of level 1-2 were included in this study. Standardised measurements of height were performed during the clinical research assistant visit. The GLI 2012 all-age reference equations were used for sex-, age- and height-specific mean predicted values of FEV1 and FVC and calculation of z-scores to determine GLI-LLN using the GLI 2012 Microsoft Excel Sheet Calculator (Global Lung Function Initiative, www.lungfunction.org). 


\section{Outcomes and variables}

All-cause mortality and time to first unplanned hospitalisation after baseline were used as outcome measurements. In addition to age and sex, other potential confounding variables were included in the statistical analysis as follows: smoking status (never, previous or current smoker), number of nonrespiratory comorbidities (unweighted disease count including anaemia, Parkinson's disease, arthritis, arthrosis, osteoporosis, cancer, depression, hypertension, diabetes, angina pectoris, myocardial infarction, cardiomyopathy, transient ischaemic attacks, cerebrovascular accidents, peripheral arterial disease, decompensated heart failure, valvular disease, thyroid dysfunction, renal failure, hyperlipidaemia and atrial fibrillation), high-sensitivity C-reactive protein (hsCRP), N-terminal pro-brain natriuretic peptide (NT-proBNP) and estimated glomerular filtration rate (eGFR). The GPs reported the recorded status of smoking and morbidities at baseline. Blood samples were collected in the morning and serum samples were stored frozen at $-80^{\circ} \mathrm{C}$ until analysis. Serum NT-proBNP levels were measured with Dade-Dimension Xpand (Siemens, Deerfield, IL, USA). Serum creatinine and hsCRP were measured with UniCel DxC 800 Synchron (Beckman Coulter, Brea, CA, USA). The eGFR was estimated according to the equation from the Modification of Diet in Renal Disease Study Group [17].

\section{Statistical analysis}

Based on the two FEV1/FVC cut-offs, the study population was divided into three airflow limitation categories: 1) participants with FEV1/FVC $\geqslant 0.70$ and $\geqslant$ GLI-LLN (no airflow limitation by both cut-offs); 2) participants with FEV1/FVC $<0.70$ and $\geqslant$ GLI-LLN (airflow limitation by fixed cut-off only); 3) participants with FEV1/FVC $<0.70$ and $<$ GLI-LLN (airflow limitation by both cut-offs). There were no participants with FEV1/FVC $\geqslant 0.70$ and $<$ GLI-LLN. The baseline variables and outcomes across the three categories were compared using ANOVA for parametric data, Kruskal-Wallis test for nonparametric data and Pearson's Chi-squared test for categorical data. Descriptive statistics are presented as the mean $\pm \mathrm{SD}$ or median with interquartile range for continuous variables and as numbers with frequencies for categorical variables. Cohen's kappa coefficient was used to measure agreement between airflow limitation defined by the two cut-offs and GP-reported COPD diagnosis. Kaplan-Meier curves for all-cause mortality and hospitalisation during follow-up were plotted for airflow limitation by each cut-off, as well as the three airflow limitation categories, with log-rank tests for comparison. Cox proportional hazards regression models were used to estimate the all-cause mortality and hospitalisation hazard ratio (HR) for airflow limitation by each cut-off, with adjustment in two consecutive models using those with no airflow limitation as the reference category. Variables were first checked for multicollinearity. The category-based net reclassification improvement (NRI) was calculated for airflow limitation defined by GLI-LLN versus fixed cut-off for all-cause mortality and hospitalisation during the follow-up [18, 19]. A two-tailed probability value $\mathrm{p}<0.05$ was considered statistically significant. Statistical analysis was performed using SPSS 22.0 for Windows (SPSS Inc., Chicago, IL, USA).

\section{Results}

Baseline characteristics of the study population

In total, 567 patients (aged $85 \pm 3.9$ years, 36.9\% males) were included in the BELFRAIL cohort. Spirometry was performed by 522 participants at baseline. After exclusion of those with asthma diagnosis in the medical chart, those aged $>95$ years (for whom no GLI 2012 reference values were available) and those with level 3 and 4 spirograms, 411 participants were included in this study's analysis (fig. 1). This subset was comparable to the BELFRAIL cohort, being aged $84.6 \pm 3.4$ years and comprising $37 \%$ males. The baseline characteristics and outcomes of the total study population and the three categories based on the two cut-offs are presented in table 1.

The airflow limitation prevalence was $9.2 \%$ by the GLI-LLN and $27 \%$ by the fixed cut-off. $17.8 \%$ of the participants had airflow limitation by fixed cut-off only (FEV1/FVC $<0.70$ and $\geqslant$ GLI-LLN). There was no statistically significant difference between the airflow limitation categories with regard to age, number of comorbidities or levels of hsCRP, NT-proBNP or eGFR. Participants with airflow limitation by both cut-offs were more often male, taller, smokers/ex-smokers and with lower body mass index compared with those with no airflow limitation by both cut-offs. The GPs reported 37 (9\%) participants with COPD at baseline and only 17 (46\%) of these had airflow limitation by both cut-offs. Agreement between airflow limitation and GP-reported COPD diagnosis was higher for the GLI-LLN cut-off, but Cohen's kappa coefficients were $<0.70$ for both cut-offs (table 2 ).

\section{Prediction of all-cause mortality and hospitalisation}

Data on all-cause mortality were available for all participants and on hospitalisation for 406 participants. During $2.93 \pm 0.21$ years, $203(50 \%)$ had at least one unplanned hospitalisation and, at $5.12 \pm 0.22$ years' follow-up, 164 (39.9\%) participants had died. The absolute numbers of deaths and times to first 
hospitalisation for each of the three categories based on the two cut-offs are presented in table 1. For either cut-off, those with airflow limitation had higher mortality than those with no airflow limitation (figs 2 and 3), but after adjustment only airflow limitation by GLI-LLN had a statistically significant higher risk, with HR 2.10 (95\% CI 1.30-3.38; p=0.002) (table 3).

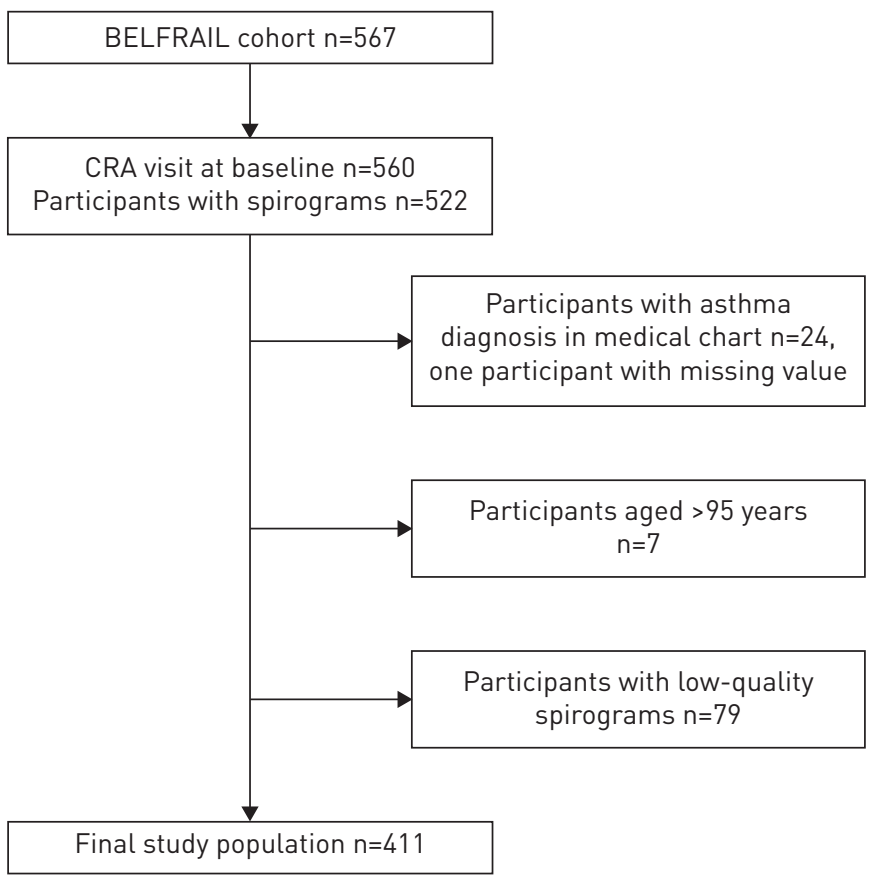

FIGURE 1 Flowchart of the selection of the study population. CRA: clinical research assistant.

TABLE 1 Baseline characteristics and outcomes of the total population and across categories based on fixed and Global Lungs Initiative lower limit of normal (GLI-LLN) ${ }^{\#}$ cut-offs for forced expiratory volume in $1 \mathrm{~s}$ (FEV1)/forced vital capacity (FVC)

Total population $\quad \mathrm{FEV}_{1} / \mathrm{FVC} \geqslant 0.70$
and $\geqslant G L I-L L N$

\begin{tabular}{l}
\hline Subjects \\
Age years \\
Males \\
Height $\mathrm{cm}$ \\
BMI $\mathrm{kg} \cdot \mathrm{m}^{-2}$ \\
Smoker/ex-smoker \\
Comorbidities \\
hsCRP $\mathrm{mg} \cdot \mathrm{dL}^{-1}$ \\
eGFR $\mathrm{mL}^{-1} \mathrm{~min}^{-1}$ per $1.73 \mathrm{~m}^{2}$ \\
NT-proBNP pg $\cdot \mathrm{mL}^{-1}$ \\
FEV1 L \\
FVC L \\
FEV1/FVC \\
COPD diagnosis in \\
medical chart \\
Mortality at 5 years \\
Time to first unplanned \\
hospitalisation \\
\end{tabular}

$125(30.4)$

$5(3-7)$

$0.17(0.07-0.40)$

$62.6(48.8-77.2)$

$195.1(97.8-531.4)$

$1.7 \pm 0.6$

$2.4 \pm 0.8$

$0.73 \pm 0.11$

37 (9)

$158.5 \pm 8.6$

$28.0 \pm 4.9$

67 (22.3)

$5(3-6)$

$0.18(0.08-0.38)$

$62.8(49.7-77.5)$

192.5 (91.5-466.8)

$1.8 \pm 0.6$

$2.3 \pm 0.7$

$0.78 \pm 0.05$

8 (2.7)

$$
\begin{gathered}
110(36.7) \\
2.1 \pm 1.0
\end{gathered}
$$

$2.0 \pm 1.0$
FEV $1 / F V C<0.70$ $<$ GLI-LLN

$\geqslant$ GLI-LLN $\quad<$ GLI-LLN

73

$84.8 \pm 3.3$

35 (47.9)

$161.6 \pm 8.6$

$26.0 \pm 4.2$

29 (39.7)

4 (2.5-7.5)

$0.12(0.05-0.38)$

$62.7(44.0-73.1)$

$193.1(107.6-589.3)$

$1.8 \pm 0.6$

$2.7 \pm 0.9$

$0.65 \pm 0.10$

12 (16.4)

38

$85.0 \pm 3.1$

$29(76.3)$

$164.4 \pm 10.7$

$24.43 \pm 4.16$

29 (76.3)

$4.5(3-6)$

$0.20(0.07-0.45)$

$60.2(47.1-82.8)$

$257.6(107.3-689.1)$

$1.3 \pm 0.5$

$2.6 \pm 0.9$

$0.51 \pm 0.08$

17 (44.7)

29 (39.7)
25 (65.8)

$1.7 \pm 1.1$ p-value

$0.73^{f}$

$<0.001^{\# \#}$

$<0.001^{f}$

$<0.001^{f}$

$<0.001^{\# \#}$

$0.97^{\text {ๆก }}$

0.19 ๆा

$0.50^{f}$

$0.92^{\text {11ๆ }}$

$<0.001^{f}$

$<0.001^{f}$

$<0.001^{f}$

$<0.001^{\# \#}$

$<0.05^{\# \#}$

$0.08^{f}$

Data are presented as $\mathrm{n}$, mean $\pm \mathrm{SD}, \mathrm{n}(\%)$ or median (interquartile range), unless otherwise stated. BMI: body mass index; hsCRP: high-sensitivity C-reactive protein; eGFR: estimated glomerular filtration rate; NT-proBNP: N-terminal pro-brain natriuretic peptide; COPD: chronic obstructive pulmonary disease. \#: 5th percentile of Global Lungs Initiative 2012 z-scores distribution; ": number of nonrespiratory comorbidities reported by the general practitioner; ${ }^{+}$: estimated according to the equation from the Modification of Diet in Renal Disease Study

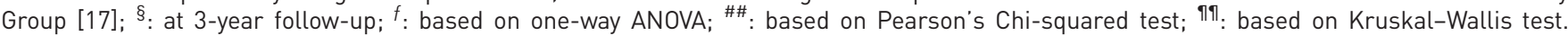
There were no missing values for the baseline variables except for hsCRP $(n=8)$, eGFR $(n=10)$ and NT-proBNP $(n=7)$. 
TABLE 2 Frequency of airflow limitation by the two cut-offs for forced expiratory volume in $1 \mathrm{~s}$ (FEV1)/forced vital capacity (FVC) and degree of agreement with general practitioner-reported chronic obstructive pulmonary disease (COPD) diagnosis

\begin{tabular}{|c|c|c|c|c|c|}
\hline & \multirow[t]{2}{*}{ Subjects } & \multicolumn{2}{|c|}{$\mathrm{FEV}_{1} / \mathrm{FVC}<0.70$} & \multicolumn{2}{|c|}{ FEV $1 / F V C<G L I-L L N$} \\
\hline & & Positive & Negative & Positive & Negative \\
\hline With COPD diagnosis & 37 & 29 & 8 & 17 & 20 \\
\hline No COPD diagnosis & 374 & 82 & 292 & 21 & 353 \\
\hline Total & 411 & 111 & 300 & 38 & 373 \\
\hline Kappa coefficient $(95 \% \mathrm{CI})$ & & \multicolumn{2}{|c|}{$0.30(0.19-0.39)$} & \multicolumn{2}{|c|}{$0.40(0.24-0.54)$} \\
\hline p-value & & \multicolumn{2}{|c|}{$<0.001$} & \multicolumn{2}{|c|}{$<0.001$} \\
\hline
\end{tabular}

Data are presented as n, unless otherwise stated. GLI-LLN: Global Lungs Initiative lower limit of normal, 5 th percentile of Global Lungs Initiative 2012 z-scores distribution.

The proportion of participants with a first hospitalisation was higher in those with airflow limitation by both cut-offs, but the difference was not statistically significant (figs 2 and 3).

The participants with airflow limitation by fixed cut-off only (FEV1/FVC $<0.70$ and $\geqslant$ GLI-LLN) did not have a higher number of deaths or proportion with hospitalisation compared with those with no airflow limitation by both cut-offs, while those with airflow limitation by GLI-LLN only (FEV1/FVC $\geqslant 0.70$ and $<$ GLI-LLN) had a significantly higher number of both deaths and proportion with hospitalisation (fig. 4).

Based on the total NRI and its components, the use of the GLI-LLN cut-off worsened the classification for $17.7 \%$ of the dead participants and $19.2 \%$ of those with at least one hospitalisation, but improved it for $17.8 \%$ of those alive and $16.8 \%$ of those with no hospitalisation during the follow-up (table 4 ). In total, there was no significant net loss for all-cause mortality and hospitalisation when using the GLI-LLN cut-off compared with the fixed one (table 4).

We also tested how the results with GLI 2012 equations compared against extrapolating from the US National Health and Nutrition Examination Survey (NHANES) III equations for our population. We found a very good level of agreement between the NHANES III and GLI 2012 LLN of FEV1/FVC (kappa coefficient $0.892, \mathrm{p}<0.001$ ), while airflow limitation based on the NHANES III LLN had a smaller adjusted HR for 5-year mortality (1.88, 95\% CI 1.19-2.97) compared with airflow limitation based on GLI 2012 LLN (2.10, 95\% CI 1.30-3.38).
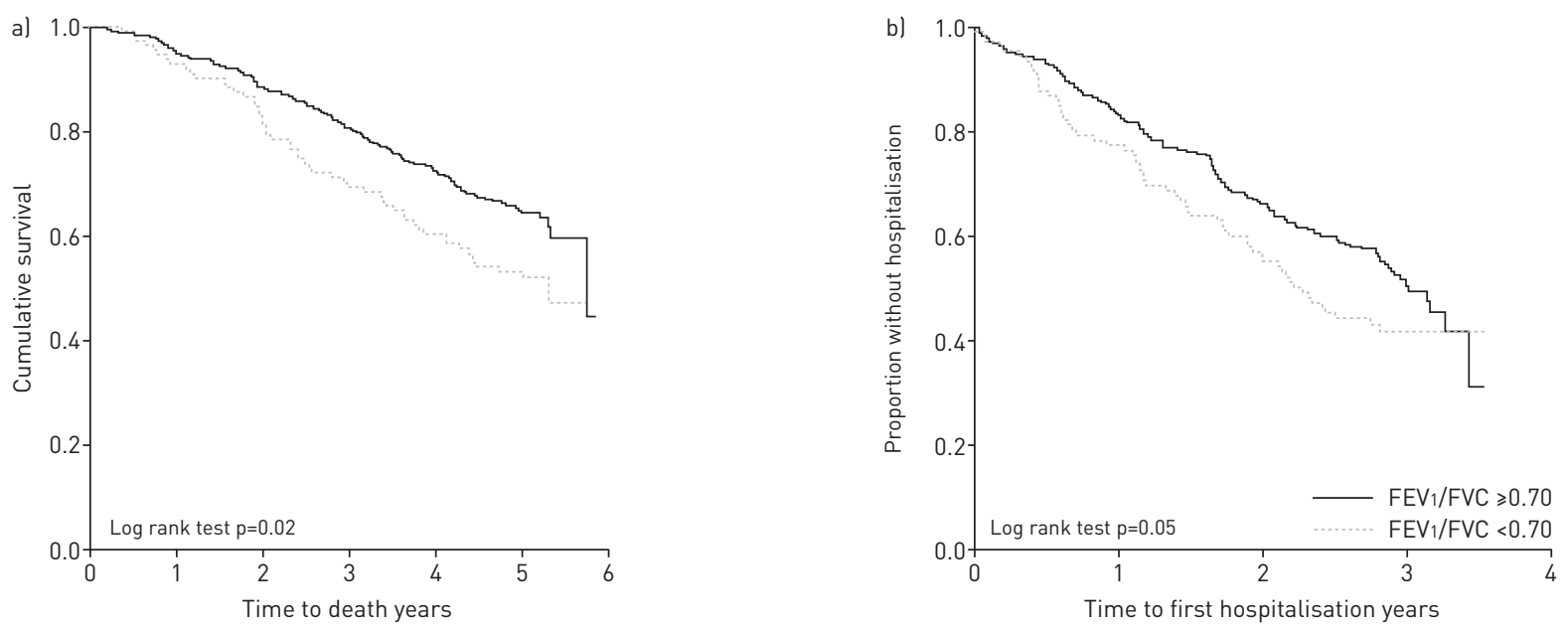

$\begin{array}{lllcccc}\text { Number at risk } & & & & & & \\ \mathrm{FEV}_{1} / \mathrm{FVC} \geqslant 0.70 & 300 & 284 & 264 & 241 & 215 & 144 \\ \mathrm{FEV} 1 / \mathrm{FVC}<0.70 & 111 & 102 & 90 & 76 & 68 & 39\end{array}$
Number at risk
$\mathrm{FEV} 1 / \mathrm{FVC} \geqslant 0.70$
$F E V_{1} / F V C<0.70$

$\begin{array}{lccc}290 & 234 & 182 & 31 \\ 108 & 81 & 56 & 10\end{array}$

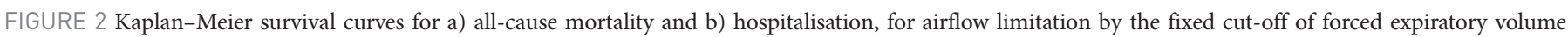
in $1 \mathrm{~s}\left(\mathrm{FEV}_{1}\right) /$ forced vital capacity (FVC). 

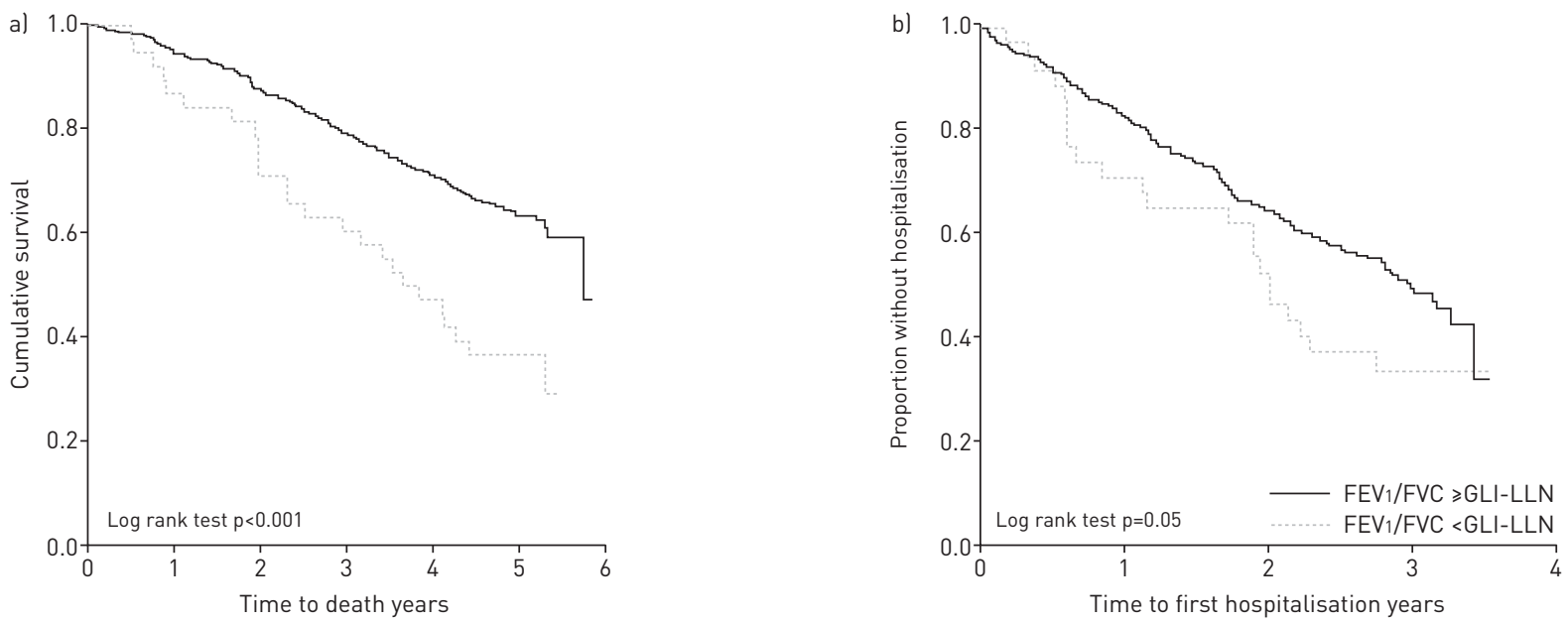

Number at risk

FEV $1 / F V C \geqslant G L I-L L N ~ 373 \quad 353 \quad 326 \quad 295 \quad 264 \quad 173$

$\begin{array}{lllllll}\mathrm{FEV}_{1} / \mathrm{FVC}<\mathrm{GLI}-\mathrm{LLN} & 38 & 33 & 28 & 22 & 19 & 10\end{array}$

Number at risk

$\mathrm{FEV}_{1} / \mathrm{FVC} \geqslant \mathrm{GLI}$-LLN $362 \quad 291 \quad 223 \quad 37$

$\mathrm{FEV}_{1} / \mathrm{FVC}<\mathrm{GLI}-\mathrm{LLN} \quad 36 \quad-24 \quad-15-42$

FIGURE 3 Kaplan-Meier survival curves for a) all-cause mortality and b) hospitalisation, for airflow limitation by the Global Lungs Initiative lower limit of normal (GLI-LLN) cut-off of forced expiratory volume in $1 \mathrm{~s}$ (FEV1)/forced vital capacity (FVC).

TABLE 3 Multivariable Cox proportional hazard analysis for all-cause mortality and airflow limitation defined by the two cut-offs

FEV $1 / F V C<0.70^{\#}$

FEV $1 /$ FVC $<$ GLI-LLN"

\begin{tabular}{|c|c|c|c|c|c|c|}
\hline & & \\
\hline & Unadjusted & Model $1^{+}$ & Model $2^{\S}$ & Unadjusted & Model $1^{+}$ & Model $2^{\S}$ \\
\hline $\mathrm{FEV}_{1} / \mathrm{FVC}$ & $1.50(1.08-2.08)^{*}$ & $1.38(0.98-1.95)$ & $1.38(0.98-1.97)$ & $2.27(1.48-3.47)^{* * *}$ & $2.16(1.35-3.44)^{* *}$ & $2.10(1.30-3.38)^{* *}$ \\
\hline Age & & $1.12(1.08-1.17)^{* * *}$ & $1.10(1.05-1.15)^{* * *}$ & & $1.12(1.08-1.17)^{* * *}$ & ${ }^{*} 1.10(1.05-1.15)^{* * *}$ \\
\hline Sex male & & $1.00(0.68-1.49)$ & $1.02(0.69-1.50)$ & & $1.03(0.69-1.53)$ & $1.06(0.72-1.58)$ \\
\hline Smoker/ex-smoker & & $1.09(0.72-1.66)$ & $1.07(0.72-1.60)$ & & $0.97(0.63-1.50)$ & $0.94(0.61-1.45)$ \\
\hline Comorbidities & & & $1.08(1.02-1.14)^{* *}$ & & & $1.09(1.03-1.15)^{* *}$ \\
\hline hsCRP $>0.3 \mathrm{mg} \cdot \mathrm{dL}^{-1}$ & & & $1.30(0.93-1.81)$ & & & $1.27(0.92-1.77)$ \\
\hline NT-proBNP highest tertile & & & $1.77(1.25-2.49) * *$ & & & $1.72(1.22-2.42)^{* *}$ \\
\hline eGFR $<45 \mathrm{~mL} \cdot \mathrm{min}^{-1}$ & & & $1.30(0.89-1.89)$ & & & $1.34(0.92-1.95)$ \\
\hline
\end{tabular}

FEV1: forced expiratory volume in $1 \mathrm{~s}$; FVC: forced vital capacity; GLI-LLN: Global Lungs Initiative lower limit of normal, 5th percentile of Global Lungs Initiative 2012 z-scores distribution; hsCRP: high-sensitivity C-reactive protein; NT-proBNP: N-terminal pro-brain natriuretic peptide; eGFR: estimated glomerular filtration rate according to the equation from the Modification of Diet in Renal Disease Study Group [17]. ${ }^{\#}$ : reference category $\mathrm{FEV}_{1} / \mathrm{FVC} \geqslant 0.70$; ${ }^{\text {? }}$ : reference category $\mathrm{FEV} / \mathrm{FVC} \geqslant \mathrm{GLI}-\mathrm{LLN}^{+}{ }^{+}$: adjusted for age, sex and smoking status; ${ }^{\S}$ : model 1 adjusted for number of nonrespiratory comorbidities reported by the general practitioner, hsCRP $>0.3 \mathrm{mg} \cdot \mathrm{dL}^{-1}$, highest sex-specific tertile of NT-proBNP and eGFR $<45 \mathrm{~mL} \cdot \mathrm{min}^{-1}{ }^{*}: \mathrm{p}<0.05 ;{ }^{* *}: \mathrm{p}<0.01 ;{ }^{* * *}: \mathrm{p}<0.001$.

\section{Discussion}

Main findings in relation to previous studies

In a cohort of very old adults, using the 5th percentile of the z-scores derived from the recent all-age GLI 2012 reference values as a cut-off for the FEV1/FVC produced much lower airflow limitation prevalence compared with the fixed cut-off, with both cut-offs having no good agreement with the GP-reported COPD. Participants with airflow limitation by either cut-off had a statistically significant higher risk for all-cause mortality but not for hospitalisation. After adjustment, only airflow limitation by GLI-LLN had a statistically significant risk for all-cause mortality. Those with airflow limitation by fixed cut-off only (discordant group) did not show a statistically significant higher risk for all-cause mortality and hospitalisation compared with those with no airflow limitation by both cut-offs. Based on total NRI, the use of GLI-LLN as a cut-off for airflow limitation causes no significant loss in predicting all-cause mortality or hospitalisation.

Previous studies have reported overdiagnosis of airflow limitation in older adults when using the fixed cut-off of FEV1/FVC versus the age- and sex-specific LLN, yet these studies did not use the GLI 2012 


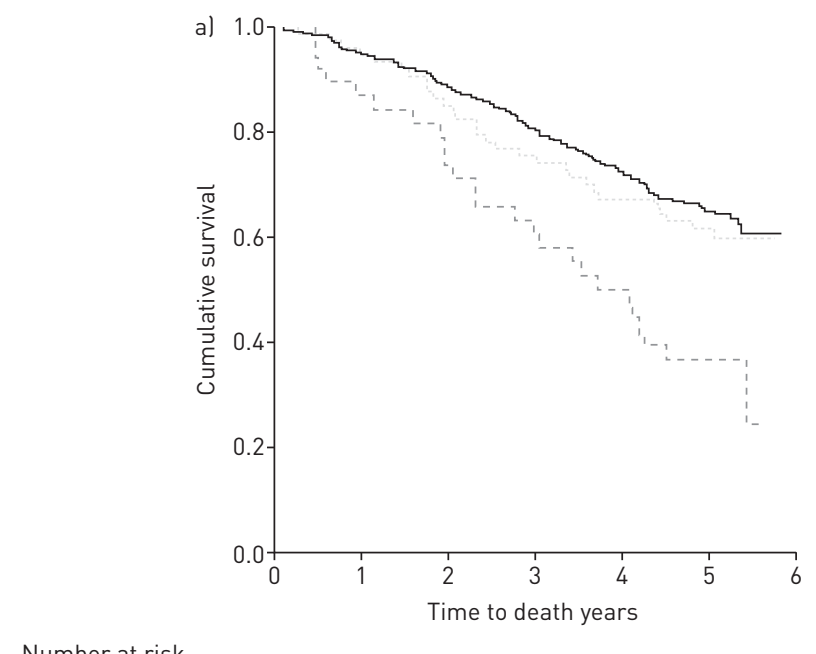

\begin{tabular}{|c|c|c|c|c|c|c|}
\hline Number at risk & & & & & & \\
\hline $\begin{array}{r}\mathrm{FEV}_{1} / \mathrm{FVC} \geqslant 0.70 \\
\text { and } \geqslant G L I-L L N\end{array}$ & 300 & 284 & 264 & 241 & 215 & 144 \\
\hline $\begin{array}{c}\mathrm{FEV}_{1} / \mathrm{FVC}<0.70 \\
\text { but } \geqslant \mathrm{GLI}-\mathrm{LLN}\end{array}$ & 73 & 69 & 62 & 54 & 49 & 29 \\
\hline $\mathrm{FEV}_{1} / \mathrm{FVC}<0.70$ & 38 & 33 & 28 & 22 & 19 & 10 \\
\hline
\end{tabular}

Number at risk

$\mathrm{FEV}_{1} / \mathrm{FVC} \geqslant 0.70$

and $\geqslant G L I-L L N$

$\mathrm{FEV} / \mathrm{FVC}<0.70$ but $\geqslant G L I-L L N$

$\mathrm{FEV}_{1} / \mathrm{FVC}<0.70$ and $<$ GLI-LLN

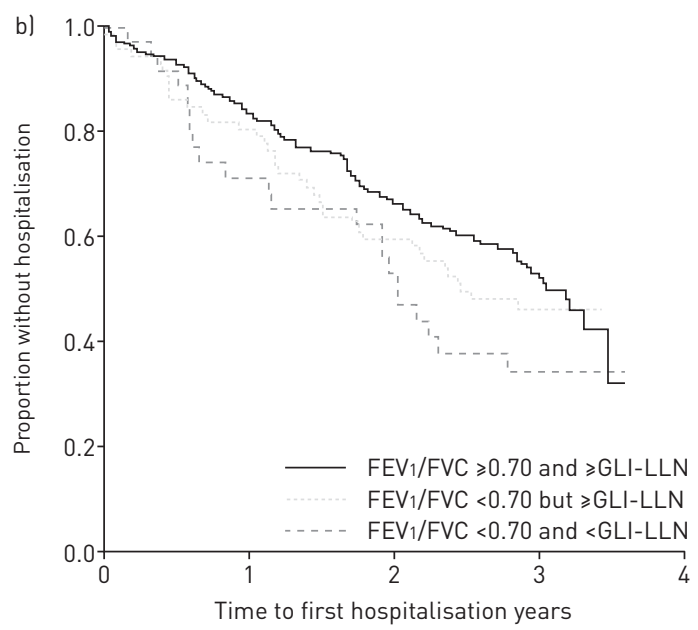

$\begin{array}{llll}290 & 234 & 182 & 31 \\ 72 & 57 & 41 & 6 \\ 36 & 24 & 15 & 4\end{array}$

FIGURE 4 Kaplan-Meier survival curves for a) all-cause mortality and b) hospitalisation, for the three groups of airflow limitation based on both the Global Lungs Initiative lower limit of normal (GLI-LLN) cut-off and the fixed cut-off of forced expiratory volume in $1 \mathrm{~s}$ (FEV1)/forced vital capacity (FVC). Pair-wise log rank test for mortality: $\mathrm{FEV} 1 / \mathrm{FVC}<0.70$ but $\geqslant$ GLI-LLN versus $\mathrm{FEV} 1 / \mathrm{FVC} \geqslant 0.70$ and $\geqslant$ GLI-LLN $\mathrm{p}=0.51 ; \mathrm{FEV} 1 / \mathrm{FVC}<0.70$ and $<\mathrm{GLI}-\mathrm{LLN}$ versus $\mathrm{FEV} 1 / \mathrm{FVC} \geqslant 0.70$ and $\geqslant$ GLI-LLN $\mathrm{p}<0.001 ; \mathrm{FEV} 1 / \mathrm{FVC}<0.70$ and $<$ GLI-LLN versus $\mathrm{FEV} 1 / \mathrm{FVC}<0.70$ but $\geqslant$ GLI-LLN $\mathrm{p}=0.01$. Pair-wise $\log$ rank test for hospitalisation: FEV1/FVC $<0.70$ but $\geqslant$ GLI-LLN versus FEV1/FVC $\geqslant 0.70$ and $\geqslant$ GLI-LLN $p=0.24$; FEV1/FVC $<0.70$ and $<$ GLI-LLN versus FEV $1 /$ FVC $\geqslant 0.70$ and $\geqslant$ GLI-LLN $p=0.04$; FEV $1 /$ FVC $<0.70$ and $<$ GLI-LLN versus $\mathrm{FEV} 1 / \mathrm{FVC}<0.70$ but $\geqslant$ GLI-LLN $\mathrm{p}=0.30$.

\begin{tabular}{|c|c|c|c|c|c|}
\hline & All & Reclassified up & Reclassified down ${ }^{+}$ & NRI $(95 \%$ CI) \% & p-value \\
\hline \multicolumn{6}{|l|}{ Mortality } \\
\hline Dead & 164 & 0 & 29 & $-17.7(-24.2--12.1)$ & $<0.01$ \\
\hline Alive & 247 & 0 & 44 & $17.8(13.3-23.1)$ & $<0.01$ \\
\hline Total & 411 & & & $0.001(-0.08-0.08)^{\S}$ & 0.99 \\
\hline \multicolumn{6}{|c|}{ Hospitalisation } \\
\hline With & 203 & 0 & 39 & $-19.2(-25.2--14)$ & $<0.001$ \\
\hline Without & 203 & 0 & 34 & 16.8 (11.9-22.5) & $<0.001$ \\
\hline Total & 406 & & & $-0.02(-0.10-0.05)^{\S}$ & 0.63 \\
\hline
\end{tabular}

Data are presented as $\mathrm{n}$, unless otherwise stated. ${ }^{\#}$ : 5th percentile of Global Lungs Initiative 2012 z-scores distribution; ": if no airflow limitation by fixed cut-off, but airflow limitation by GLI-LLN; ${ }^{+}$: if airflow limitation by fixed cut-off, but no airflow limitation by GLI-LLN; ${ }^{\S}$ : total NRI expressed as a proportion [19].

reference equations and few have used the LMS approach $[20,21]$. To our knowledge, there are no data available yet on the prevalence of airflow limitation using the GLI-LLN for people aged $\geqslant 80$ years, as the GLI 2012 reference equations have been made available only recently [7, 10]. Their application in clinical populations so far shows a $16-23 \%$ decrease of airflow limitation prevalence in adults aged $>55$ years compared with the fixed cut-off [22]. In two samples of community-dwelling people aged 65-80 years, airflow limitation prevalence was $13.2-13.8 \%$ for LLN (using the LMS approach but based on other reference equations) versus $36.7-37.7 \%$ for fixed cut-off $[9,20]$. Our findings in very old adults and using the GLI 2012 equations are similar (9\% for GLI-LLN versus 27\% for fixed cut-off). 
In our cohort, only $46 \%$ of GP-reported COPD diagnoses were confirmed by both cut-offs and $32 \%$ by the fixed cut-off only, while $22 \%$ had no airflow limitation by either cut-off. Although GP-reported COPD diagnosis is not the gold standard, it is the real-life situation. In another study, even when using diagnosis by a panel of lung disease experts, $17 \%$ of cases were missed by the fixed and $28 \%$ by the LLN cut-off, while airflow limitation by the fixed cut-off had higher agreement with the expert panel's diagnosis [23]. In our study, neither of the cut-offs had good agreement with the GP-reported COPD diagnosis, but we had a non-clinical study population with a small number of participants with COPD diagnosis $(\mathrm{n}=37)$, no information on the diagnostic process, as well as a different age group (aged $\geqslant 80$ years). Spirometry is required for COPD diagnosis $[2,4]$, yet most COPD cases in primary or secondary care are not confirmed by spirometry especially with increasing age [24]. Besides spirometry, COPD diagnosis requires assessment of respiratory symptoms, risk factors and comorbidities $[2,4]$, as even similar degrees of airflow limitation have different effects on symptoms, comorbidities and outcomes $[25,26]$. In very old adults, COPD diagnosis becomes even more complex, as respiratory symptoms such as dyspnoea may be assigned to ageing and common comorbidities such as heart failure [27]. Thus, a comprehensive diagnostic approach, which recognises the heterogeneity and clinical phenotypes of COPD and includes spirometry, needs to be applied $[2,3,25,26]$.

As concluded in two recent systematic reviews, due to a lack of gold standard for COPD diagnosis it is not possible to state which FEV1/FVC cut-off for airflow limitation is best, and longitudinal studies are needed to evaluate association with adverse health outcomes $[12,21]$. In line with previous findings in large population-based studies of people aged 65-80 years (using the LMS approach but based on other reference equations) $[9,20]$, in our cohort of very old adults airflow limitation defined by GLI-LLN was associated with increased HR for mortality even after adjustment for number of comorbidities (out of 21), systemic inflammatory marker levels (hsCRP) and a heart failure marker (NT-proBNP adjusted for eGFR levels), which have not been included in previous studies. Heart failure is a common comorbidity in very old adults that can influence the spirometry parameters, causing either restrictive or obstructive changes, and is also associated with mortality [28]. NT-proBNP has been found to be valuable in ruling out heart failure in older adults (including those with COPD) and to be a predictor of mortality in very old adults $[29,30]$. We corrected NT-proBNP for renal dysfunction (eGFR $<45 \mathrm{~mL} \cdot \mathrm{min}^{-1}$ ) [31].

Some studies have reported that individuals with FEV1/FVC $<0.70$ and $\geqslant$ LLN have higher adjusted risk for all-cause mortality compared with those with no airflow limitation by both cut-offs, cautioning that the use of the LLN cut-off would miss individuals at risk [32, 33]. However, these findings have been contested [34,35], and other studies have reported no significant difference in mortality as well as COPD-related outcomes (COPD hospitalisation, exacerbations, FEV1 decline) [10-12]. Studies on COPD phenotypes also show that the discordant cases in older adults mostly have milder airflow limitation and better survival $[25,36]$. In line with these findings, in our cohort of very old adults, those with FEV1/FVC $<0.70$ and $\geqslant$ GLI-LLN had no statistically significant higher all-cause mortality and hospitalisation compared with those with no airflow limitation by both cut-offs. In addition, we found that, although GLI-LLN was less sensitive (events NRI, i.e. dead/with hospitalisation) and more specific (non-events NRI, i.e. alive/without hospitalisation) compared with the fixed cut-off, in total its use caused no significant loss in predicting either all-cause mortality or hospitalisation.

\section{Strengths and limitations of this study}

This study has several strengths. It is based on a large heterogeneous population, representative of very old adults in Belgium, with follow-up for around 5 years [14]. The majority (92\%) of the original cohort performed spirometry and almost 79\% achieved quality spirograms according to ATS/ERS criteria. Rigorous quality control of spirometry performance and interpretation based on the ATS/ERS quality criteria were followed and various confounders were included in the analysis, covering demographics, smoking status and number of comorbidities, as well as markers of systemic inflammation and heart failure. The use of pre-bronchodilator values introduces the risk for overestimation of irreversible airflow limitation by 25-29\% [37]. This limitation is partly reduced by the exclusion of participants with GP-reported asthma diagnosis. Pre- and post-bronchodilator values have also been found to have similar accuracy for mortality prediction [38]. All-cause mortality and hospitalisation were used as outcomes instead of COPD-related outcomes. In our population of very old adults with several comorbidities, it is difficult to assign these adverse events to COPD, which itself is associated with comorbidities, and many COPD deaths/hospitalisations are assigned to cardiovascular diseases and lung cancer [9]. The exclusion of participants with low-quality spirometry and those aged $>95$ years (the age limit of the GLI 2012 reference values) is also a limitation, but this caused no statistically significant differences in baseline and outcome variables. We did not exclude individuals with restrictive pulmonary dysfunction as we had no measurements of total lung volumes [6]. As a restrictive pattern in spirometry (reduced FVC with normal $\mathrm{FEV} 1 / \mathrm{FVC}$ ) has been found to be associated with mortality, this might have influenced our results [20,32]. However, we found no statistically 
significant differences in frequencies of FVC $<80 \%$ predicted and FVC $<$ GLI-LLN between the three airflow limitation categories. We also had similar results after excluding those with $\mathrm{FVC}<80 \%$ predicted and FVC $<$ GLI-LLN from those with FEV1/FVC $\geqslant 0.70$ and FEV1/FVC $\geqslant$ GLI-LLN, respectively.

\section{Implications for future research and practice}

The LMS-derived LLN cut-off of FEV1/FVC is preferred over the fixed cut-off of 0.70 , as it takes into account the age-related decline in FEV1/FVC, variability of spirometry values and skewness of reference data, and has also been shown to be clinically relevant $[8,10,12]$. The recent GLI 2012 all-age reference values allow its use for people aged 80-95 years and are easy to access and apply [7]. In our representative cohort of very old adults in Belgium, using the GLI-LLN reduced airflow limitation prevalence compared with the fixed cut-off of FEV1/FVC and did not miss individuals with significantly higher all-cause mortality or hospitalisation. After adjustment for a number of confounders including a heart failure biomarker, only airflow limitation by GLI-LLN was an independent predictor for 5-year all-cause mortality. More longitudinal studies are needed on the clinical validation of the GLI-LLN using COPD-related adverse outcomes and focusing on people aged $\geqslant 80$ years, which is a growing age group worldwide [10]. Correct identification of airflow limitation in very old adults is important as it may reduce inappropriate COPD diagnosis and use of COPD medications, along with their side-effects [10].

It has recently been suggested that, for very old adults, extrapolating from the NHANES III equations may be better than using GLI 2012 for predicting survival from FEV1 [39]. However, we found that GLI 2012 gave better survival predictions than NHANES III when used to identify airflow limitation from FEV1/ FVC in our cohort of very old adults. Although there is still room for improvement of the GLI 2012 reference values for those aged $\geqslant 80$ years $[7,39]$, the use of these global all-age and multi-ethnic reference equations in future studies would help the comparison across research findings, as well as their translation into practice to optimise diagnosis and treatment of respiratory diseases, especially in very old adults.

\section{Acknowledgements}

The authors would like to thank the following GPs from Belgium who made possible this study by recruiting their patients: Etienne Baijot (Beauraing), Pierre Leclercq (Pondrôme), Baudouin Demblon (Wellin), Daniel Simon (Rochefort), Daniel Vanthuyne (Celles), Yvan Mouton (Godinne), Louis-Philippe Docquier (Maffe), Tanguy Dethier (Ciney), Patricia Eeckeleers (Leignon), Jean-Paul Decaux (Dinant), Christian Fery (Dinant), Pascale Pierret (Heure), Paul-Emile Blondeau (Beauraing), Baudry Gubin (Beauraing), Jacques Guisset (Wellin), Quentin Gillet (Mohiville), Arlette Germay (Houyet), Jan Craenen (Hoeilaart), Luc Meeus (Hoeilaart), Herman Docx (Hoeilaart), Ann Van Damme (Hoeilaart), Sofie Dedeurwaerdere (Hoeilaart), Bert Vaes (Hoeilaart), Stein Bergiers (Hoeilaart), Bernard Deman (Hoeilaart), Edmond Charlier (Overijse), Serge Tollet (Overijse), Eddy Van Keerberghen (Overijse), Etienne Smets (Overijse), Yves Van Exem (Overijse), Lutgart Deridder (Overijse), Jan Degryse (Oudergem), Katrien Van Roy (Oudergem), Veerle Goossens (Tervuren), Herman Willems (Overijse) and Marleen Moriau (Bosvoorde).

\section{References}

1 Mannino DM, Buist AS. Global burden of COPD: risk factors, prevalence, and future trends. Lancet 2007; 370: 765-773.

2 Global Initiative for Chronic Obstructive Lung Disease (GOLD). Global Strategy for the Diagnosis, Management and Prevention of Chronic Obstructive Pulmonary Disease (Updated 2015). GOLD, 2015. Available from www. goldcopd.org

3 Quanjer PH. Correctly defining criteria for diagnosing chronic obstructive pulmonary disease matters. Am J Respir Crit Care Med 2014; 189: 230.

4 Celli BR, MacNee W. Standards for the diagnosis and treatment of patients with COPD: a summary of the ATS/ ERS position paper. Eur Respir J 2004; 23: 932-946.

5 Pellegrino R, Viegi G, Brusasco V, et al. Interpretative strategies for lung function tests. Eur Respir J 2005; 26 : 948-968.

6 Stanojevic S, Wade A, Stocks J. Reference values for lung function: past, present and future. Eur Respir J 2010; 36: $12-19$.

7 Quanjer PH, Stanojevic S, Cole TJ, et al. Multi-ethnic reference values for spirometry for the 3-95-yr age range: the global lung function 2012 equations. Eur Respir J 2012; 40: 1324-1343.

8 Stanojevic S, Wade A, Stocks J, et al. Reference ranges for spirometry across all ages: a new approach. Am J Respir Crit Care Med 2008; 177: 253-260.

9 Vaz Fragoso CA, Concato J, McAvay G, et al. The ratio of FEV1 to FVC as a basis for establishing chronic obstructive pulmonary disease. Am J Respir Crit Care Med 2010; 181: 446-451.

10 Vaz Fragoso CA, Gill TM, McAvay G, et al. Respiratory impairment in older persons: when less means more. Am J Med 2013; 126: 49-57.

11 Akkermans RP, Biermans M, Robberts B, et al. COPD prognosis in relation to diagnostic criteria for airflow obstruction in smokers. Eur Respir J 2014; 43: 54-63.

12 van Dijk WD, Gupta N, Tan WC, et al. Clinical relevance of diagnosing COPD by fixed ratio or lower limit of normal: a systematic review. COPD 2014; 11: 113-120.

13 Dept of Economic and Social Affairs, Population Division. World Population Ageing, 2009. New York, United Nations, 2009.

14 Vaes $\mathrm{B}$, Pasquet $\mathrm{A}$, Wallemacq $\mathrm{P}$, et al. The BELFRAIL $\left(\mathrm{BF}_{\mathrm{C} 80+}\right)$ study: a population-based prospective cohort study of the very elderly in Belgium. BMC Geriatr 2010; 10: 39. 
15 Degryse J, Buffels J, Van Dijck Y, et al. Accuracy of office spirometry performed by trained primary-care physicians using the MIR Spirobank hand-held spirometer. Respiration 2012; 83: 543-552.

16 Miller MR, Hankinson J, Brusasco V, et al. Standardisation of spirometry. Eur Respir J 2005; 26: 319-338.

17 Levey AS, Bosch JP, Lewis JB, et al. A more accurate method to estimate glomerular filtration rate from serum creatinine: a new prediction equation. Modification of Diet in Renal Disease Study Group. Ann Intern Med 1999; 130: 461-470.

18 Pencina MJ, D’Agostino RB Sr, Steyerberg EW. Extensions of net reclassification improvement calculations to measure usefulness of new biomarkers. Stat Med 2011; 30: 11-21.

19 Leening MJ, Vedder MM, Witteman JC, et al. Net reclassification improvement: computation, interpretation, and controversies: a literature review and clinician's guide. Ann Intern Med 2014; 160: 122-131.

20 Vaz Fragoso CA, Gill TM, McAvay G, et al. Respiratory impairment and mortality in older persons: a novel spirometric approach. J Investig Med 2011; 59: 1089-1095.

21 Mohamed Hoesein FA, Zanen P, Lammers JW. Lower limit of normal or $\mathrm{FEV}_{1} / \mathrm{FVC}<0.70$ in diagnosing COPD: an evidence-based review. Respir Med 2011; 105: 907-915.

22 Quanjer PH, Brazzale DJ, Boros PW, et al. Implications of adopting the Global Lungs Initiative 2012 all-age reference equations for spirometry. Eur Respir J 2013; 42: 1046-1054.

23 Güder G, Brenner S, Angermann CE, et al. GOLD or lower limit of normal definition? A comparison with expert-based diagnosis of chronic obstructive pulmonary disease in a prospective cohort-study. Respir Res 2012; 13: 13.

24 Arne M, Lisspers K, Ställberg B, et al. How often is diagnosis of COPD confirmed with spirometry? Respir Med 2010; 104: 550-556.

25 Burgel PR, Paillasseur JL, Caillaud D, et al. Clinical COPD phenotypes: a novel approach using principal component and cluster analyses. Eur Respir J 2010; 36: 531-539.

26 Rennard SI, Vestbo J, Agusti A. What is chronic obstructive pulmonary disease anyway? Continua, categories, cut points, and moving beyond spirometry. Am J Respir Crit Care Med 2013; 187: 1036-1037.

27 Coultas DB, Davis K. Hidden realities of COPD among the very old. In: Bellia V, Antonelli Incalzi R, eds. Respiratory Diseases in the Elderly. ERS Monogr 2009; 43: 77-89.

28 Güder G, Rutten FH. Comorbidity of heart failure and chronic obstructive pulmonary disease: more than coincidence. Curr Heart Fail Rep 2014; 11: 337-346.

29 Rutten FH, Moons KG, Cramer MJ, et al. Recognising heart failure in elderly patients with stable chronic obstructive pulmonary disease in primary care: cross sectional diagnostic study. BMJ 2005; 331: 1379.

30 Vaes B, Delgado V, Bax J, et al. Diagnostic accuracy of plasma NT-proBNP levels for excluding cardiac abnormalities in the very elderly. BMC Geriatr 2010; 10: 85 .

31 deFilippi CR, Seliger SL, Maynard S, et al. Impact of renal disease on natriuretic peptide testing for diagnosing decompensated heart failure and predicting mortality. Clin Chem 2007; 53: 1511-1519.

32 Mannino DM, Buist AS, Vollmer WM. Chronic obstructive pulmonary disease in the older adult: what defines abnormal lung function? Thorax 2007; 62: 237-241.

33 Mannino DM, Diaz-Guzman E. Interpreting lung function data using 80\% predicted and fixed thresholds identifies patients at increased risk of mortality. Chest 2012; 141: 73-80.

34 Miller MR. Lung function data interpretation. Chest 2012; 141: 832-833.

35 Quanjer PH, Pretto JJ, Brazzale DJ, et al. Grading the severity of airways obstruction: new wine in new bottles. Eur Respir J 2014; 43: 505-512.

36 Burgel PR, Roche N, Paillasseur JL, et al. Clinical COPD phenotypes identified by cluster analysis: validation with mortality. Eur Respir J 2012; 40: 495-496.

37 Vollmer WM, Gislason T, Burney P, et al. Comparison of spirometry criteria for the diagnosis of COPD: results from the BOLD study. Eur Respir J 2009; 34: 588-597.

38 Mannino DM, Diaz-Guzman E, Buist S. Pre- and post-bronchodilator lung function as predictors of mortality in the Lung Health Study. Respir Res 2011; 12: 136.

39 Miller MR, Thinggaard M, Christensen $\mathrm{K}$, et al. Best lung function equations for the very elderly selected by survival analysis. Eur Respir J 2014; 43: 1338-1346. 